Supporting Information for Organometallics

\title{
About the crystal structure of $[\operatorname{Rh}((S, S)$-DIPAMP $)((Z)$-2-benzoylamino-3- (3,4-dimethoxyphenyl)-methyl acrylate) $\mathrm{BF}_{4}-$ Major or minor catalyst-substrate complex?
}

\author{
T. Schmidt, W. Baumann*, H.-J. Drexler, A. Arrieta, D. Heller* \\ Leibniz-Institut für Organische Katalyse an der Universität Rostock e.V. \\ Albert-Einstein-Strasse 29a, D-18059 Rostock, Phone +49 3811281183 Fax: +49 3811281 \\ 5000 \\ E-mail: detlef.heller@ifok-rostock.de \\ wolfgang.baumann@ ifok-rostock.de \\ H. Buschmann, \\ ESTEVE, Av. Mare de Deu de Montserrat 221, ES-08041 Barcelona, Spain
}




\section{Additional NMR analyses}

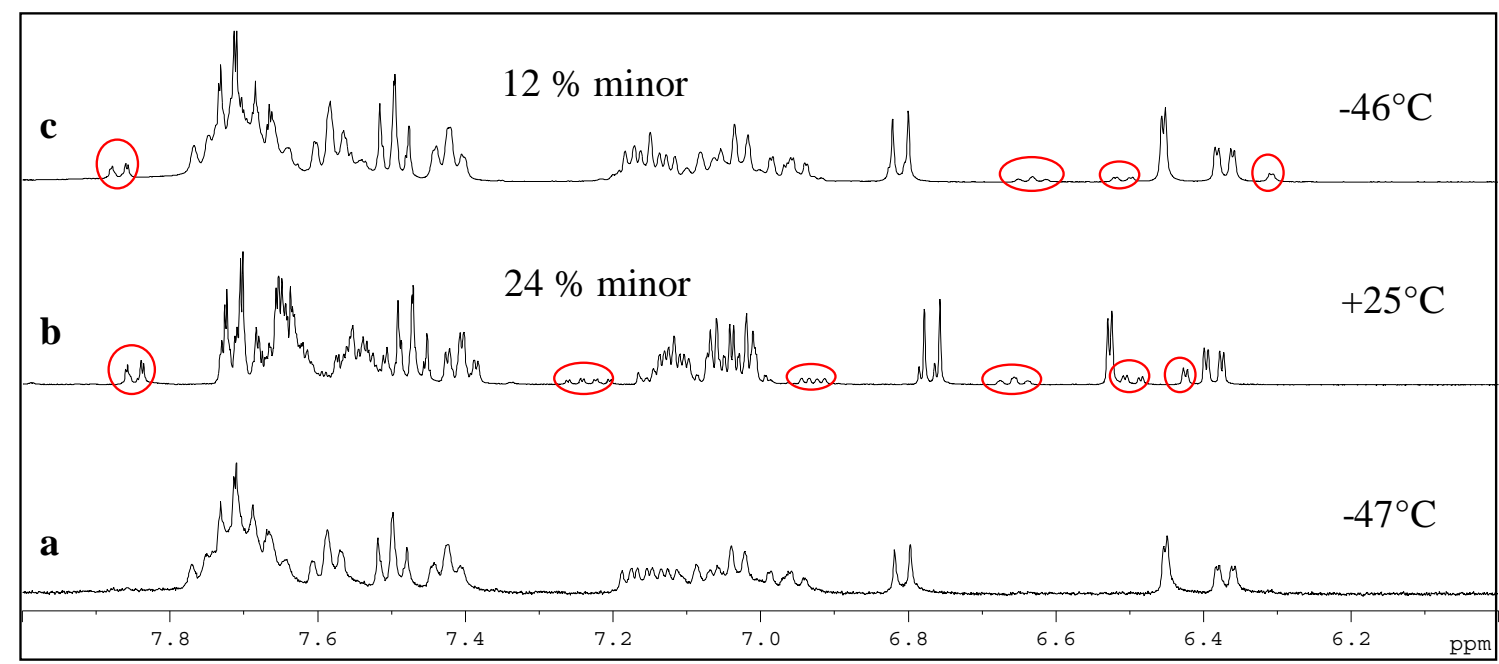

Figure supp. inf. 1: Proton spectra (aryl group area) of the dissolved single crystals of $\left[\mathrm{Rh}((S, S)\right.$-DIPAMP)((Z)-2-benzoylamino-3-(3,4-dimethoxyphenyl)-methyl acrylate) $] \mathrm{BF}_{4}$ at various temperatures.

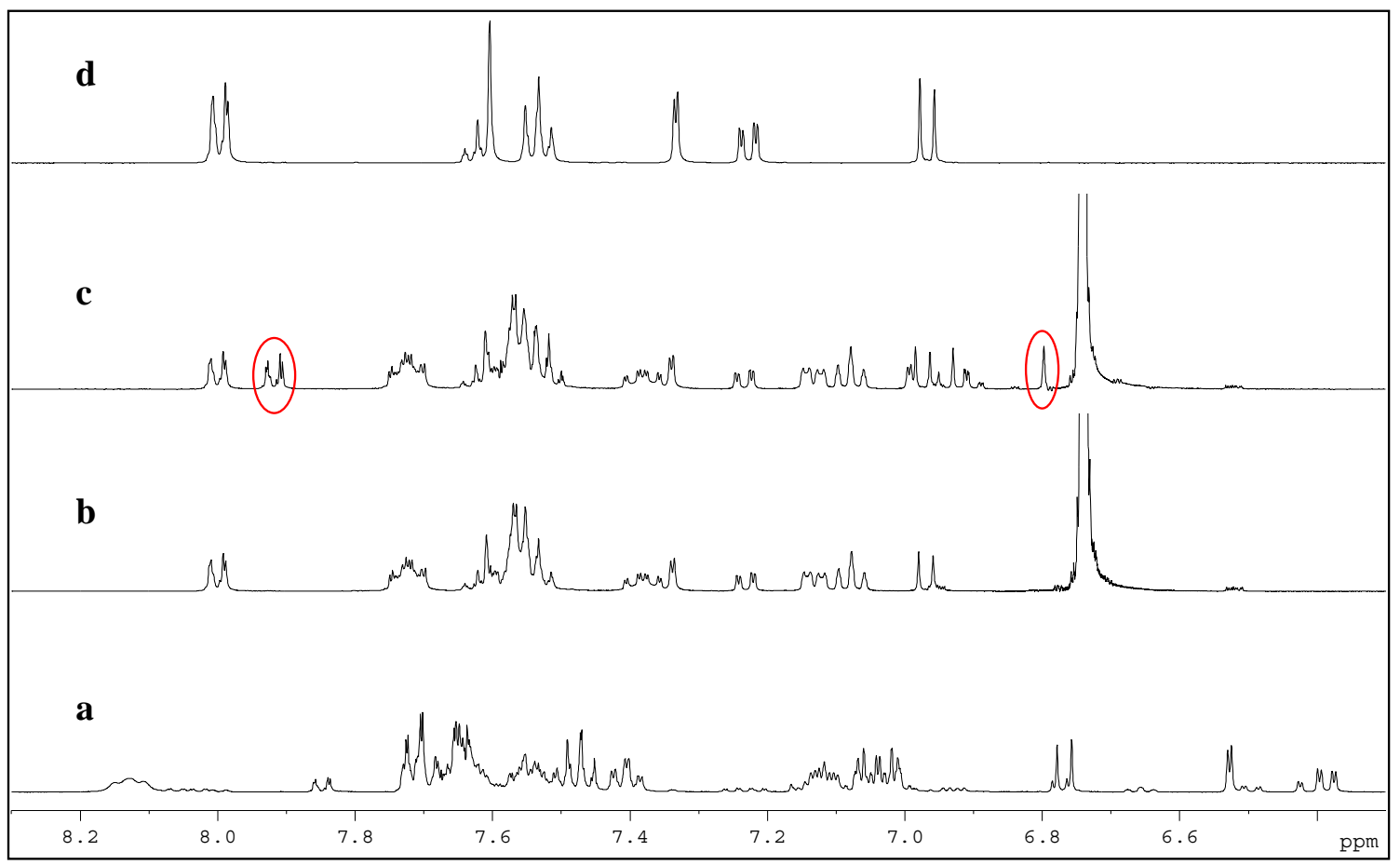

Figure supp. inf. 2: Proton spectra (aryl group area) to exclude $Z / E$-isomerisation. a) dissolved single crystals; b) addition of NBD; c) addition of $(E)$-2-benzoylamino-3-(3,4dimethoxy-phenyl)-methyl acrylate; d) (Z)-2-benzoylamino-3-(3,4-dimethoxy-phenyl)-methyl acrylate. 


\section{Crystallographic Experimental Section}

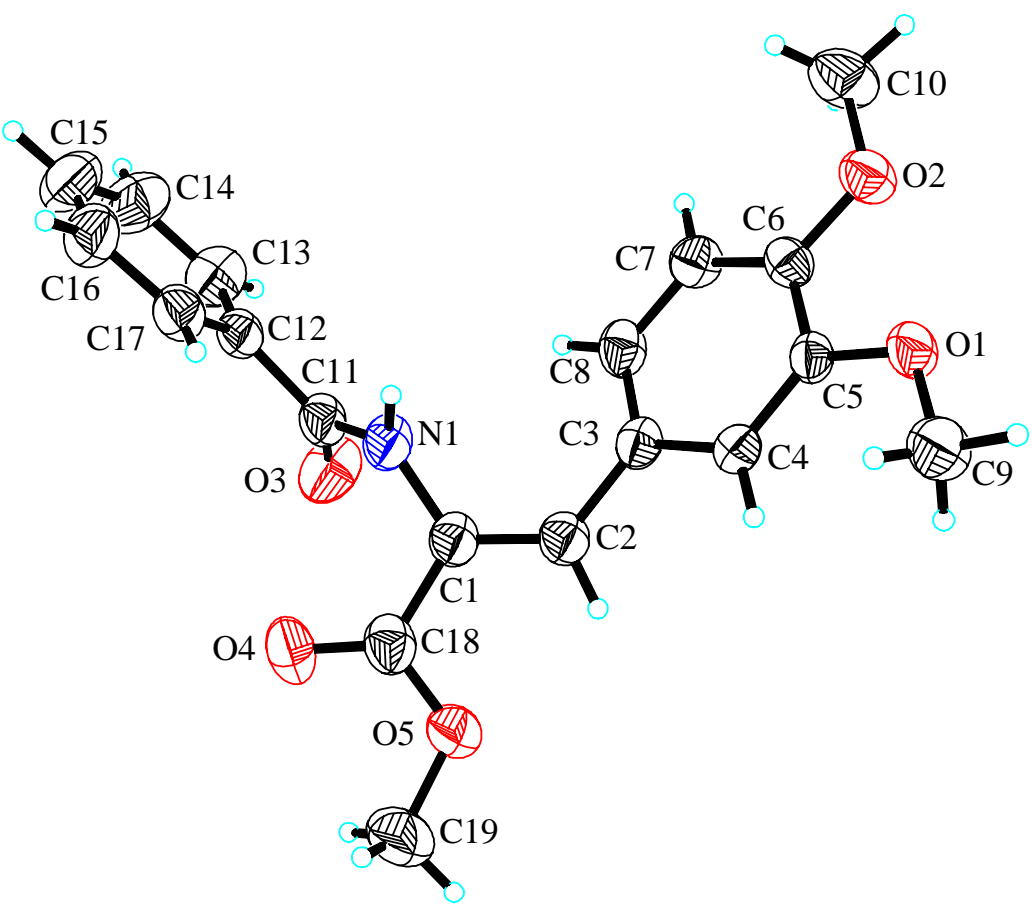

Figure supp. inf. 3: Solid-state molecular structure of (Z)-2-benzoylamino-3-(3,4dimethoxyphenyl)-methyl acrylate (ORTEP, $50 \%$ probability ellipsoids).

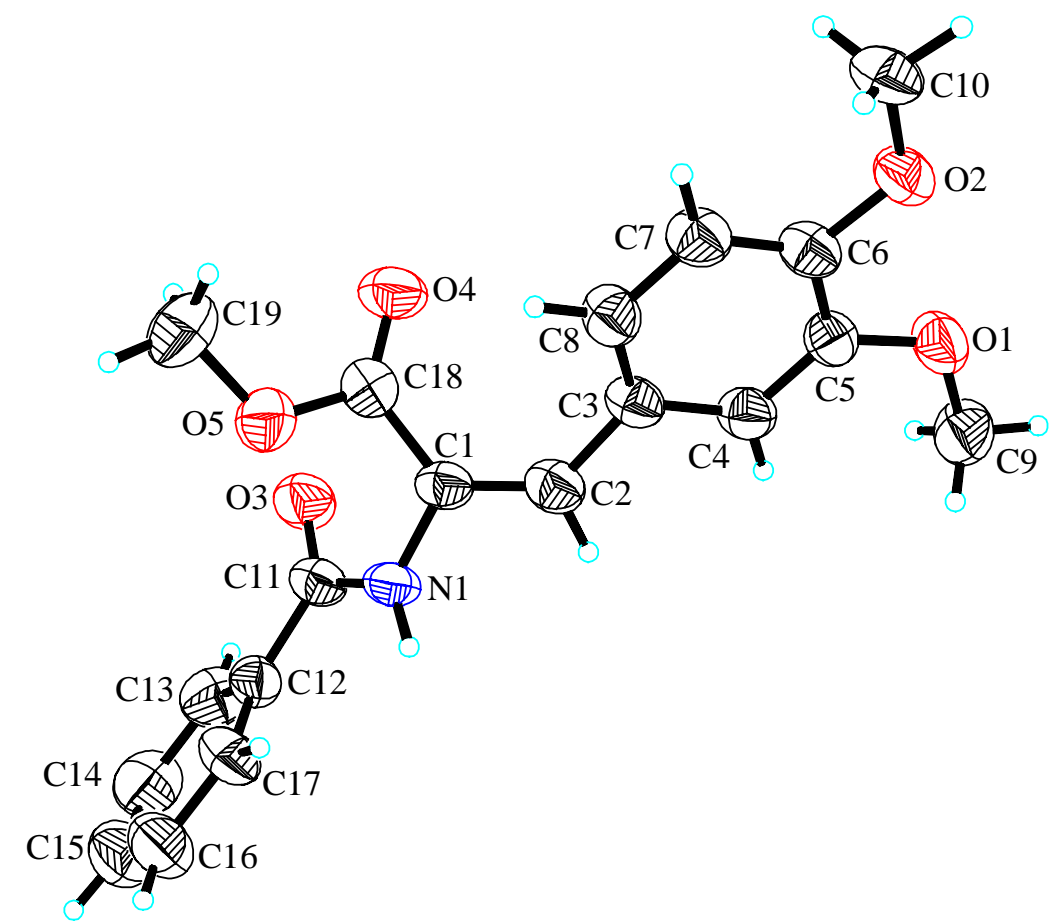

Figure supp. inf. 4: Solid-state molecular structure of (E)-2-benzoylamino-3-(3,4dimethoxyphenyl)-methyl acrylate (ORTEP, $50 \%$ probability ellipsoids). 
Diffraction data were collected on a STOE-IPDS diffractometer $\left[\lambda\left(\mathrm{Mo}_{\mathrm{O}} \mathrm{K}_{\alpha}\right)=0.71073 \AA\right]$. The structures were solved by direct methods (SHELXS-97 (Sheldrick, 1997)) and refined by full matrix least square techniques against $F^{2}$ (SHELXL-97). XP (Siemens Analytical X-ray Instruments, Inc.) was used for structure representations. As observation criterion I > $2 \mathrm{~s}(\mathrm{I})$ was used.

The nonhydrogen atoms were refined anisotropically. The hydrogen atoms were placed into theoretical positions and were refined by using the riding model.

\begin{tabular}{|c|c|c|}
\hline Compound & Z-Isomer $\cdot \mathrm{MeOH}$ & E-Isomer \\
\hline Empirical formula & $\mathrm{C}_{20} \mathrm{H}_{23} \mathrm{NO}_{6}$ & $\mathrm{C}_{19} \mathrm{H}_{19} \mathrm{NO}_{5}$ \\
\hline Formula weight & 373.39 & 341.35 \\
\hline Crystal system & monoclinic & monoclinic \\
\hline Space group & $\mathrm{P} 2{ }_{1} / \mathrm{n}$ & $\mathrm{P} 2{ }_{1} / \mathrm{c}$ \\
\hline $\mathrm{a} \quad[\AA]$ & $10.081(2)$ & $14.184(3)$ \\
\hline$[\AA]$ & $16.466(3)$ & $12.060(2)$ \\
\hline [A] & $12.008(2)$ & $9.940(2)$ \\
\hline$\left[{ }^{\circ}\right]$ & $103.01(3)$ & $92.11(3)$ \\
\hline $\mathrm{V} \quad\left[\AA^{3}\right]$ & $1942.1(7)$ & $1699.2(6)$ \\
\hline $\mathrm{d}_{\mathrm{c}}\left[\mathrm{Mg} / \mathrm{m}^{3}\right]$ & 1.277 & 1.334 \\
\hline $\mathrm{Z}$ & 4 & 4 \\
\hline$\mu\left[\mathrm{mm}^{-1}\right]$ & 0.095 & 0.097 \\
\hline $\mathrm{F}(000)$ & 792 & 720 \\
\hline Crystal size $[\mathrm{mm}]$ & $0.5 \times 0.4 \times 0.3$ & $0.5 \times 0.05 \times 0.05$ \\
\hline Temperature $[\mathrm{K}]$ & 293 & 293 \\
\hline Scan range $(2 \Theta)\left[^{\circ}\right]$ & $2.14-26.14$ & $2.22-22.50$ \\
\hline Index range (hkl) & $-12 / 12,-19 / 20,-14 / 14$ & $-15 / 15,-12 / 12,-10 / 10$ \\
\hline Reflection collected & 12344 & 7499 \\
\hline Independent reflections & 3799 & 2137 \\
\hline Observed reflections & 1738 & 1059 \\
\hline Parameter & 246 & 227 \\
\hline $\mathrm{R} 1(2 \sigma(\mathrm{I}))$ & 0.0459 & 0.0556 \\
\hline R1 (all data) & 0.1219 & 0.1144 \\
\hline wR2 (all data) & 0.1086 & 0.1395 \\
\hline Goodness of fit & 0.791 & 0.813 \\
\hline $\begin{array}{l}\text { Largest difference peak and hole } \\
(\mathrm{e} / \AA)\end{array}$ & $0.290 /-0.270$ & $0.191 /-0.211$ \\
\hline
\end{tabular}

Table supp. inf. 1: Crystallographic and refinement data for the $Z$-isomer and the $E$-isomer 
Crystallographic data (excluding structure factors) for the structures reported in this paper have been deposited at the Cambridge Crystallographic Data Centre as supplementary publication no. CCDC-251115 for the Z-Isomer and CCDC-251116 for the E-Isomer. Copies of the data can be obtained free of charge on application to The Director, CCDC, 12 Union Road, Cambridge, CB21EZ, UK (fax: int. code + (1223) 336-033; e-mail: deposit@ccdc.cam.ac.uk; web, www: http://www.ccdc.cam.ac.uk). 